Article

\title{
Remote Evaluation of Rotational Velocity Using a Quadrant Photo-Detector and a DSC Algorithm
}

\author{
Xiangkai Zeng ${ }^{*}{ }^{\dagger}$, Zhixiong Zhu ${ }^{\dagger}$ and Yang Chen \\ School of Optoelectronic Information, Chongqing University of Technology, Chongqing 400054, China; \\ 13677610413@139.com (Z.Z.); chenyangbisheng@163.com (Y.C.) \\ * Correspondence: zxkai@cqut.edu.cn; Tel.: +86-136-5056-5000 \\ + These authors contributed equally to this work. \\ Academic Editor: Caterina Ciminelli \\ Received: 28 February 2016; Accepted: 20 April 2016; Published: 25 April 2016
}

\begin{abstract}
This paper presents an approach to remotely evaluate the rotational velocity of a measured object by using a quadrant photo-detector and a differential subtraction correlation (DSC) algorithm. The rotational velocity of a rotating object is determined by two temporal-delay numbers at the minima of two DSCs that are derived from the four output signals of the quadrant photo-detector, and the sign of the calculated rotational velocity directly represents the rotational direction. The DSC algorithm does not require any multiplication operations. Experimental calculations were performed to confirm the proposed evaluation method. The calculated rotational velocity, including its amplitude and direction, showed good agreement with the given one, which had an amplitude error of $\sim 0.3 \%$, and had over 1100 times the efficiency of the traditional cross-correlation method in the case of data number $N>4800$. The confirmations have shown that the remote evaluation of rotational velocity can be done without any circular division disk, and that it has much fewer error sources, making it simple, accurate and effective for remotely evaluating rotational velocity.
\end{abstract}

Keywords: remote evaluation; rotational velocity; differential subtraction correlation; quadrant photodetector; temporal-delay number

\section{Introduction}

Rotational parameters, which include angular displacement, velocity and acceleration, can describe the performance, operation states and kinetic characteristics of rotating systems [1,2], such as the vortices of fluid substances, gear transfer systems, rotational machines and fan blades, etc. The exact measurement of transient velocity is vitally important for accurately controlling and monitoring the movements of some bodies, and for acquiring fault information in machine diagnoses [3]. Thus the method of sensing rotational velocity can be used for the analyses, monitoring and control of mechanical systems, swirling gas- or fluid flow, etc.

For sensing rotational or angular velocity, there exist methods using gyroscopes, marking methods, interferometry, correlation methods, circular grating sensing and spatial filtering methods, etc. The gyroscope method using a Sagnac-effect-based integrated gyroscope with small footprint has high resolution, and can sense transient angular velocity [4-6]. However it is not suitable for remote measurement and super-low velocity, and can be affected by environmental factors $[7,8]$. The interferometry methods, including Fabry-Perot cavities and Mach-Zehnder interferometers, have complicated structures and high precision fabrication requirements [9-11]. In the correlation method, the traditional cross-correlation (TCC) is often used to process the output signals of two identical sensors in the measured region [12-14], which provides non-contact measurements with low cost for monitoring gas-solid and solid-liquid flows [15-18]. The image matching method, in which a high-speed camera captures a series of instantaneous images to calculate velocity, also belongs to 
the correlation methods [19-22]. Correlation methods are less applied for remotely sensing transient angular velocity, due to the numerous multiplication operations required. Marking methods with optical labels (plus quadrant photodiode) or Hall-effect sensors mainly sense average rotational velocities with lower resolution or direction ambiguity $[23,24]$, which can hardly measure instantaneous rotational velocity. The methods based on circular gratings like optical gratings $[25,26]$ and magnetic grids can be employed to sense the transient angular velocity of target objects with high resolution $[27,28]$. Their circular gratings must be set up as circular division disks to be coaxially mounted on the measured object, and their resolution and precision are dependant on the grating grooves. Optical gratings are easily destroyed by tiny dust particles and greasy dirt. In some cases such as those of high-speed rotating machines or when measuring gas- or fluid flows, etc., circular benchmarks cannot be mounted on the measured systems die to limitations in their structures, or their performances are possibly deteriorated. In the spatial filtering (SF) method [29], a center coefficient is obtained from the central frequencies of the output signals of two differential spatial filters. Then the rotational velocity is calculated with the central frequencies and the center coefficient, and its direction is determined by the center coefficient and one linear displacement direction. Rotational velocity measurements using the SF method are complicated, since they need to compute all the center coefficients, the central frequencies and the displacement direction.

This paper will present a novel approach for remotely evaluating transient rotational velocities with a quadrant photo-detector (QPD) and a differential subtraction correlation (DSC) algorithm proposed by us, which does not require any circular indexing plates or benchmarks. The QPD outputs four random signals, from which the amplitude and direction of the rotational velocity are obtained simultaneously by using the DSC algorithm that only needs addition and subtraction operations without any multiplication or division, which should make it faster than other correlation methods. This paper is organized as follows: Section 2 shows the rotational velocity sensing system; Section 3 describes its evaluation principle in detail; Section 4 presents some experimental calculations to verify the proposed approach, and the final section gives our conclusions.

\section{System for Sensing Rotational Velocity}

The quadrant photo-detector for sensing rotational- or angular-velocity, shown in Figure 1, is formed by four photovoltaic cells PD1, PD2, PD3 and PD4. PD1 and PD2 are arranged in parallel each other with distance $P$, which constitute a linear velocity sensor (LVS1), and output signals $S_{1}$ and $S_{2}$.

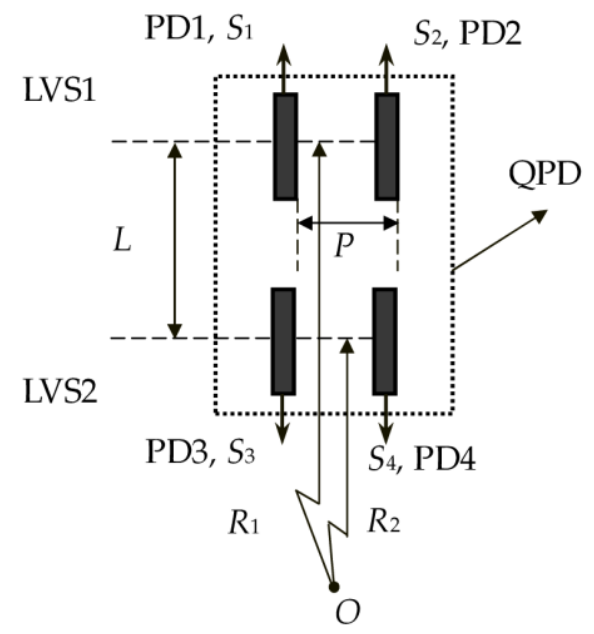

Figure 1. Structure of the QPD formed by four photovoltaic cells. 
Similarly, PD3 and PD4 are also arranged in parallel each other with the distance $P$, which lead to another linear velocity sensor (LVS2), and output signals $S_{3}$ and $S_{4}$. The LVS1 and LVS2 are also aligned in parallel each other at the interval of $L$, and then form the QPD.

The rotational velocity sensing system with the QPD and the DSC algorithm is schematically illustrated in Figure 2. Suppose the terminal plane of a measured object has a rough surface, and rotates around center $O^{\prime}$ with a rotational velocity $\omega$. The surface of the terminal plane is imaged on the QPD through an optical telescope system with magnification $M$. The image of the measured object rotates around center $O$ that is the imaged point of center $O^{\prime}$, and it must cover the QPD. Thus the size of the terminal plane must be at least larger than $L / M$. The distances of center $O$ to LVS1 and LVS2 are $R_{1}$ and $R_{2}$, respectively. The output signals of the QPD are random, owing to the stochastic reflection image of the terminal plane. The output signals $S_{1}, S_{2}, S_{3}$ and $S_{4}$ will go to the data processing system (DPS) after they are amplified and converted into digital signals. In the DPS, the DSC between $S_{1}$ and $S_{2}$ is calculated and noted as DSC1, which is related to the linear velocity of moving image on PD1 and PD2. The DSC between $S_{3}$ and $S_{4}$ is also obtained and denoted as DSC2, which indicates the linear velocity of moving image on PD3 and PD4. The rotational velocity $\omega$ of the measured object can be derived from the DSC1 and DSC2.

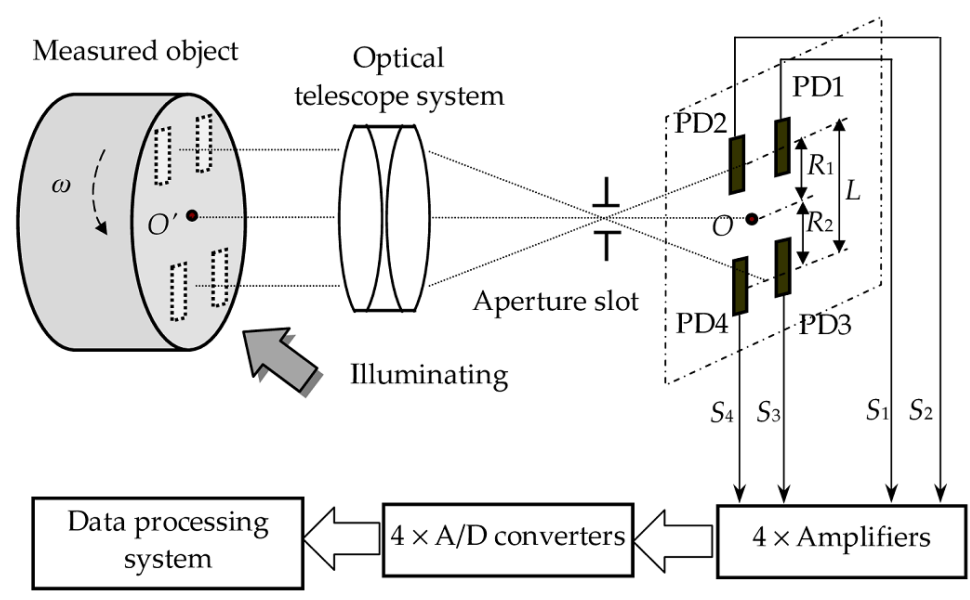

Figure 2. Schematic diagram of rotational velocity sensing system with DSC and the QPD.

\section{Principle of Evaluating Rotational Velocity with DSC}

\subsection{Image Velocity on $Q P D$}

Supposing that the terminal plane of measured object rotates around the $O^{\prime}$ in the rotational velocity of $\omega$, the terminal plane and its imaged plane are depicted in Figure 3a,b, respectively, which are viewed from the optical telescope system. LVS1', LVS2' and $O^{\prime}$ on the terminal plane are conjugated to LVS1, LVS2 and $O$ on the image plane, respectively.

In Figure $3 a, D^{\prime}{ }_{1}$ and $D^{\prime}{ }_{2}$ are two arbitrary points on the central line of the LVS1'. The interval between $O^{\prime}$ and the central line of LVS1' is $R^{\prime}{ }_{1}$, and the distances of $O^{\prime}$ to $D^{\prime}{ }_{1}$ and $D_{2}^{\prime}$ are $R^{\prime}{ }_{\mathrm{D} 1}$ and $R^{\prime}{ }_{\mathrm{D} 2}$, respectively. In Figure $3 b, D_{1}$ and $D_{2}$ are the conjugate points of $D_{1}^{\prime}$ and $D_{2}^{\prime}$, respectively, thus the distances of $O$ to $D_{1}$ and $D_{2}$ are $R_{\mathrm{D} 1}=M R_{\mathrm{D} 1}^{\prime}$ and $R_{\mathrm{D} 2}=M R_{\mathrm{D} 2}^{\prime}$, respectively. With the rotational velocity of $\omega$, the linear velocities at $D_{1}$ and $D_{2}$ are $V_{\mathrm{D} 1}=\omega M R_{\mathrm{D} 1}^{\prime}=\omega R_{\mathrm{D} 1}$ and $V_{\mathrm{D} 2}=\omega M R_{\mathrm{D} 2}^{\prime}=\omega R_{\mathrm{D} 2}$, respectively. $V_{\mathrm{D} 1}$ and $V_{\mathrm{D} 2}$ have the components $V_{\mathrm{D} 1} \times \cos \alpha$ and $V_{\mathrm{D} 2} \times \cos \beta$, respectively, in the direction of LVS1, where $\alpha$ and $\beta$ are the angles of $R_{1}$ relative to lines $O D_{1}$ and $O D_{2}$, respectively.

Meanwhile, $R_{\mathrm{D} 1} \times \cos \alpha=R_{\mathrm{D} 2} \times \cos \beta=R_{1}$, thus the image velocity $V_{1}$ on any point of the central line of the LVS1 can be obtained, such that:

$$
V_{1}=\omega R_{1}
$$




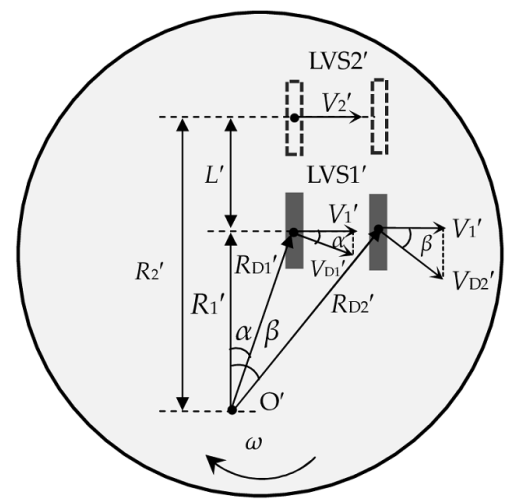

(a)

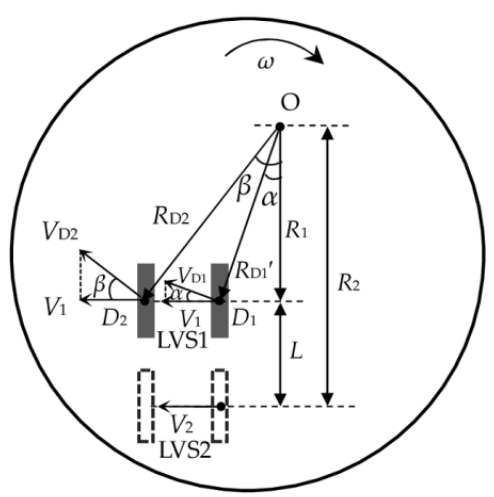

(b)

Figure 3. Relations of the rotational velocity $\omega$ with the linear velocities on (a) the terminal plane and (b) the image plane.

Similarly, we can also get the image velocity $V_{2}$ on any point of the central line of the LVS2, governed by:

$$
V_{2}=\omega R_{2}
$$

where $R_{2}$ is the interval between $O$ and the central line of LVS2. The image velocities $V_{1}$ and $V_{2}$ are insensitive to the variation of the amplification coefficient $M$ which has been hidden in $R_{1}$ and $R_{2}$ on the image plane.

\subsection{Characteristics of Output Signals of $Q P D$}

When a light source illuminates the terminal plane of the measured object that has a rough surface with finite and stochastic reflection, some light rays will be scattered by the rough surface, and then be imaged on the QPD through the optical telescope system with a high cut-off frequency in the spatial domain. The image on the QPD locating the image plane is randomly distributed, and has a wide spatial bandwidth. The output signal of each photovoltaic cell in the QPD is the integration of the randomly-reflected light intensity on its active area, which is also stochastic. Thus the QPD outputs four stochastic signals that are related to the random distribution of the rotating image, and the relations among the QPD output signals are involved with the image movement decided by the rotation of the terminal plane.

It is assumed that the central lines of LVS1 and LVS2 are $x$-axes that are parallel with PD1-PD2 and PD3-PD4, respectively. Figure 4 shows schematically the stochastic light-intensity distributions on the active areas of the QPD whose PD1-PD4 positions are also indicated in $x-y$ coordinate, where $f_{1}(x)$ and $f_{2}(x)$ are the examples of actual light-intensity distributions along the LVS1 and the LVS2, respectively, $x_{0}$ is the $x$-axial position of PD1 and PD3, $c$ is the width of the photovoltaic cells.

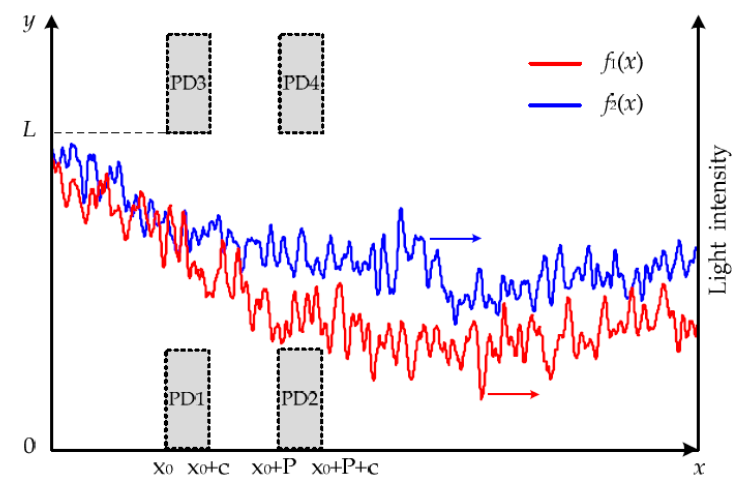

Figure 4. PD1-PD4 positions and the actual light-intensity distributions along LVS1 and LVS2. 
If only considering $x$-axial movement, the integration of the $y$-axially distributed random light at $x$-position can be taken as the whole light intensity at $x$-position, which ignores the $y$-axial factor and still is a stochastic distribution on the $x$-axis, shown as $f_{1}(x)$ or $f_{2}(x)$. When the rough surface of a measured body is imaged on the QPD, the QPD will be illuminated by the randomly distributed light that is regarded as only an $x$-axial distribution, and the output signals of the PD1-PD4 are the integrations of the light intensity distributions $f_{1}(x)$ or $f_{2}(x)$ in the $x$-axial widths of the PD1-PD4, respectively. If the measured body is rotating, its scattering light will be randomly changed, and then the QPD will output signals $S_{1}, S_{2}, S_{3}$ and $S_{4}$ that are stochastic in time domain.

If the LVS1 (PD1) is at position $x_{0}$, its output signals $S_{1}$ and $S_{2}$ will be the integrations of $f_{1}(x)$ in the regions of PD1 and PD2, respectively, and they have the relation $S_{2}\left(x_{0}\right)=S_{1}\left(x_{0}-P\right)$. If the image on the LVS1 is moving in the image velocity $V_{1}$, then the space-time relation $x_{0}=V_{1} t$ allows $S_{1}\left(x_{0}\right)$ and $S_{2}\left(x_{0}\right)$ to become time-domain signals $S_{1}(t)$ and $S_{2}(t)$, described by:

$$
\left.\begin{array}{c}
S_{1}(t)=\int_{x_{0} / V_{1}}^{\left(x_{0}+c\right) / V_{1}} f_{1}\left(V_{1} t\right) V_{1} d t \\
S_{2}(t)=\int_{\left(x_{0}+P\right) / V_{1}}^{\left(x_{0}+V_{1}\right.} f_{1}\left(V_{1} t\right) V_{1} d t
\end{array}\right\}
$$

The active-area factor of the photovoltaic cells cannot be ignored, thus the integrations in $S_{1}(t)$ and $S_{2}(t)$ will filter some high-frequency components of the light intensity distributions on PD1 and PD2, which actually function as low-pass filters that can remove much relevant characteristic information. When the measured object revolves around $O^{\prime}$, its image on PD1 and PD2 can be divided into three parts: I, II and III, as illustrated in Figure 5.

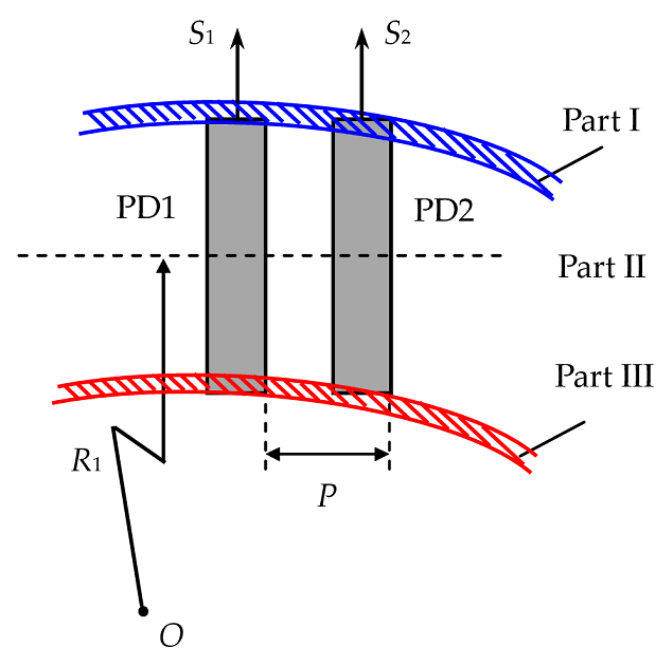

Figure 5. Region division of the rotational image on the LVS1.

Part I and Part III are labeled by blue slanted lines and red slanted lines, respectively, and Part II is the region between Part I and Part III. Part II with the largest area makes principal contribution to $S_{1}(t)$ and $S_{2}(t)$, and Parts I and III can be ignored because of their much less contributions. On other hand, the image of Part II on PD1 will be on PD2 after the delay time $\tau_{1}=P / V_{1}$. Thus Equation (3) indicates that $S_{2}(t)$ is approximately equal to the temporally delayed signal of $S_{1}(t)$ with the delay time of $\tau_{1}$, which can be written as:

$$
S_{2}(t) \approx S_{1}\left(t-\tau_{1}\right)
$$

where $\tau_{1}>0$ represents the movement direction from left to right, and $\tau_{1}<0$ represents that from right to left. Once $\tau_{1}$ is ascertained, the image velocity $V_{1}$ on the LVS1 will be determined by:

$$
V_{1}=\omega R_{1}=P / \tau_{1}
$$


Like the analogous $S_{1}(t)$ and $S_{2}(t), S_{4}(t)$ is also approximately equal to the temporally delayed signal of $S_{3}(t)$ with the delay time of $\tau_{2}$, which is expressed to be $S_{4}(t) \approx S_{3}\left(t-\tau_{2}\right)$ where $\tau_{2}=P / V_{2}$ is the time delay of $S_{3}(t) . \tau_{2}>0$ also indicates the movement direction from left to right, and $\tau_{2}<0$ indicates that from right to left. Once $\tau_{2}$ is obtained, the image velocity $V_{2}$ on the LVS 2 will be also calculated with:

$$
V_{2}=\omega R_{2}=P / \tau_{2}
$$

Based on the above descriptions, we can establish the features of the temporal output signals of the QPD: (1) they are random, corresponding to the spatially-stochastic reflection of the terminal plane; (2) the signals $S_{2}(t)$ and $S_{4}(t)$ are approximately interrelated to $S_{1}(t)$ and $S_{3}(t)$, respectively; (3) some high-frequency information consisting of more useful characteristics has been filtered, which is a disadvantage to correlation analysis. If the delay times $\tau_{1}$ and $\tau_{2}$ are obtained, Equations (5) and (6) may let the rotational velocity $\omega$ be calculated. According to the abovementioned features, the DSC algorithm is exploited to derive the delay times from the output signals.

\subsection{Differential Subtraction Correlation}

The output signals of the QPD are random and correlative, thus we also employ correlation analysis to calculate $\tau_{1}$ and $\tau_{2}$. However the conventional correlation method is low efficient, due to its multiplication operations with the complexity of about $\mathrm{O}\left(3 N \log _{2} N\right)$ or $\mathrm{O}\left(N^{2}\right)$, where $N$ is the number of sampling data. In order to fast and exactly determine the delayed time, we propose the DSC to analyze the correlation $\varphi_{1}(\tau)$ between $S_{1}(t)$ and $S_{2}(t)$, defined as:

$$
\varphi_{1}(\tau)=\int_{T_{1}}^{T_{2}}\left|\frac{d S_{1}(t-\tau)}{d t}-\frac{d S_{2}(t)}{d t}\right| d t=\int_{T 1}^{T_{2}}\left|d S_{1}(t-\tau)-d S_{2}(t)\right|
$$

where $T_{1}$ and $T_{2}$ are the start time and end time, respectively, of the integration. In practice, $S_{1}(t)$ and $S_{2}(t)$ are converted into digital signals $s_{1}(n)$ and $s_{2}(n)$, respectively, by using a multi-channel A/D converter with sampling interval $T_{0}$. Thus the DSC $\varphi_{1}(\tau)$ is discretized into $\Phi_{1}(m)$ (i.e., DSC1) as:

$$
\Phi_{1}(m)=\sum_{n=N_{1}}^{N_{2}-1}\left|s_{1}(n-m+1)-s_{1}(n-m)-s_{2}(n+1)+s_{2}(n)\right|
$$

where $N_{1}=T_{1} / T_{0}, N_{2}=T_{2} / T_{0}$ and $m=\tau / T_{0}$ are integral. If $S_{2}(t)=S_{1}\left(t-\tau_{1}\right), \Phi_{1}(m)$ will go to zero at $m=m_{1}=\tau_{1} / T_{0}$, and is much more than 0 at other $m$, where $m_{1}$ is an integer representing the temporal delay $\tau_{1}$.

Similarly, the output signals $S_{3}(t)$ and $S_{4}(t)$ of the LVS2 are converted into digital signals $s_{3}(n)$ and $s_{4}(n)$, respectively, with the same sampling interval $T_{0}$. Then $s_{3}(n)$ and $s_{4}(n)$ are sent to the DPS to calculate the discrete DSC $\Phi_{2}(m)$ (i.e., DSC2) between $s_{3}(n)$ and $s_{4}(n)$, given by:

$$
\Phi_{2}(m)=\sum_{n=N_{1}}^{N_{2}-1}\left|s_{3}(n-m+1)-s_{3}(n-m)-s_{4}(n+1)+s_{4}(n)\right|
$$

If $S_{4}(t)=S_{3}\left(t-\tau_{2}\right), \Phi_{2}(m)$ will also go to zero at $m=m_{2}=\tau_{2} / T_{0}$, and is much more than 0 at other $m$, where $m_{2}$ is an integer representing the temporal delay $\tau_{2}$.

In practice, the output signals of the QPD in this rotational velocity sensing system have the relations $S_{2}(t) \approx S_{1}\left(t-\tau_{1}\right)$ and $S_{4}(t) \approx S_{3}\left(t-\tau_{2}\right)$, which let $\Phi_{1}(m)$ and $\Phi_{2}(m)$ go to the minimums at $m=m_{1}$ and $m=m_{2}$, respectively, and they are much more than 0 at other $m$. Based on Equations (8) and (9), the DSC algorithm is then described as follows:

(1) Acquiring $s_{1}(n), s_{2}(n), s_{3}(n)$ and $s_{4}(n)$ by discretizing the output signals of the QPD with synchronous four-channel A/D converter; 
(2) Calculating the DSC1 and DSC2 at different $m$, according to Equations (8) and (9) with $s_{1}(n)$, $s_{2}(n), s_{3}(n)$ and $s_{4}(n)$;

(3) Searching the minimums of $\Phi_{1}(m)$ and $\Phi_{2}(m)$, and taking the serial numbers at the minimums of $\Phi_{1}(m)$ and $\Phi_{2}(m)$ as the temporal-delay numbers $m_{1}$ and $m_{2}$, respectively.

The temporal-delay numbers $m_{1}$ and $m_{2}$ have indicated the delay times $\tau_{1}$ and $\tau_{2}$, respectively. The proposed DSC algorithm just requires subtraction, absolute and addition operations without any multiplications or divisions, so it can facilitate the fast correlation calculation of two random signals. Furthermore, its difference operation between two adjacent data can recover high-frequency components, and can eliminate low-frequency noise and shift, and then can enlarge the differences between adjacent data, which are advantageous to improve accuracy.

\subsection{Evaluation of Rotational Velocity}

According to the DSC algorithm described before, $m_{1}$ and $m_{2}$ can be derived from $\Phi_{1}(m)$ and $\Phi_{2}(m)$ by searching the minima of $\Phi_{1}(m)$ and $\Phi_{2}(m)$, respectively. Because $\tau_{1}=m_{1} T_{0}$ and $\tau_{2}=m_{2} T_{0}$, $m_{1}>0$ and $m_{2}>0$ indicate the image movement directions from left to right on the LVS1 and the LVS2, respectively, and $m_{1}<0$ and $m_{2}<0$ indicate that from right to left on the LVS1 and the LVS2, respectively. Thus Equations (1), (2), (5) and (6) lead to the relations of the rotational velocity $\omega$ with $m_{1}$ and $m_{2}$, governed by:

$$
\begin{aligned}
& \omega R_{1}=P /\left(m_{1} T_{0}\right) \\
& \omega R_{2}=P /\left(m_{2} T_{0}\right)
\end{aligned}
$$

Now let us suppose that the LVS1 is above the LVS2. The location of the rotational center $O$ on the image plane may be between the LVS1 and the LVS2, below both the LVS1 and the LVS2, or above both the LVS1 and the LVS2, as shown in Figure 6a-c, respectively.

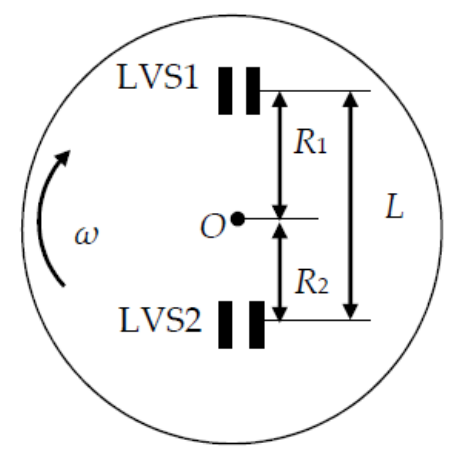

(a)

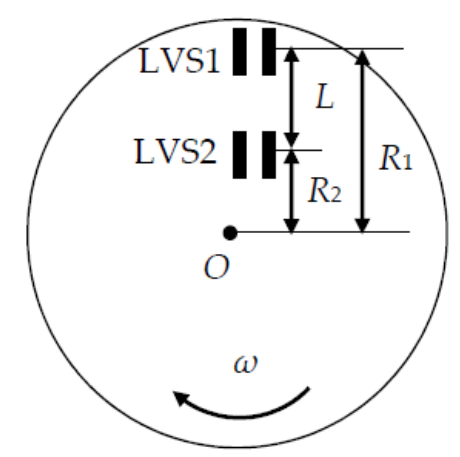

(b)

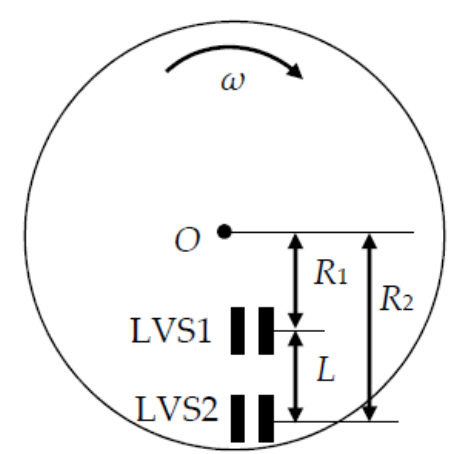

(c)

Figure 6. Locations of the rotational center $O$ relative to the LVS1 and the LVS2: (a) $O$ locates between LVS1 and LVS2; (b) $O$ is below both LVS1 and LVS2; (c) $O$ is above both LVS1 and LVS2.

The signs of $m_{1}$ and $m_{2}$ represent the image movement directions on the LVS1 and LVS2, which can be used for judging the rotational center locations and directions. In Figure 6a, the sign of $m_{1}$ is always different from that of $m_{2}$, and $m_{1}>0$ and $m_{1}<0$ demonstrate the clockwise rotational direction and the counter-clockwise one, respectively. In Figure $6 \mathrm{~b}, \mathrm{c}$, the sign of $m_{1}$ is always the same as $m_{2}$, and their rotational center locations can be discriminated by comparing $m_{1}$ to $m_{2}$, where $\left|m_{1}\right|<\left|m_{2}\right|$ and $\left|m_{1}\right|>\left|m_{2}\right|$ as indicated in Figure $6 \mathrm{~b}, \mathrm{c}$, respectively, owing to the fact $R_{2} \pm L=R_{1}$. In Figure $6 \mathrm{~b}, m_{1}>0$ and $m_{1}<0$ illustrate the clockwise rotational direction and the counter-clockwise one, respectively. In Figure $6 c, m_{1}<0$ and $m_{1}>0$ mean the clockwise rotational direction and the counter-clockwise one, respectively. At the same time, Figure 6a-c have shown $R_{1}+R_{2}=L, R_{1}-R_{2}=L$ with $\left|m_{1}\right|<$ $\left|m_{2}\right|$, and $R_{2}-R_{1}=L$ with $\left|m_{1}\right|>\mid m_{2}$, respectively, from which we can derive the rotational velocity 
$\omega$ as $\left(1 / m_{1}-1 / m_{2}\right) P /\left(L T_{0}\right)$ by using Equations (10) and (11). The sign of $\omega$ is the same as that of $m_{1}$ in the cases of Figure $6 \mathrm{a}, \mathrm{b}$, and is opposite that of $m_{1}$ in the case of Figure $6 \mathrm{c}$, which shows that $\omega>0$ and $\omega<0$ always represent the clockwise rotational direction and the counter-clockwise one, respectively. Thus in all cases of different directions and centers, the sign of $\omega$ always indicates the rotational direction, and the rotational velocity $\omega$ can be determined by:

$$
\omega=\frac{P}{L T_{0}}\left(\frac{1}{m_{1}}-\frac{1}{m_{2}}\right)
$$

Note that $\left|m_{1}\right|$ and $\left|m_{2}\right|$ are not equal to 0 in Equation (12), and must be less than $N$ at least in the DSCs. In practice, they should be less than $N / 2$, in order to accurately acquire them. Thus the better range of the rotational velocity calculated with Equation (12) should be from $2 P /\left(N T_{0} R_{\min }\right)$ to $P /\left(T_{0} R_{\max }\right)$, where $R_{\min }=\min \left(R_{1}, R_{2}\right)$ and $R_{\max }=\max \left(R_{1}, R_{2}\right)$.

The relations of $\omega$ with $m_{1}, m_{2}$, rotational center location and direction are listed in Table 1 . The rotational velocity $\omega$ calculated with Equation (12) includes the amplitude and direction of the rotational velocity on the image plane, which is corresponding to the rotational velocity of the measured object. Therefore once $m_{1}$ and $m_{2}$ are obtained, the rotational velocity consisting of its amplitude and direction will be calculated by only using Equation (12).

Table 1. The relations of $\omega$ with rotational direction, center location, $m_{1}$ and $m_{2}$.

\begin{tabular}{cccc}
\hline \multirow{2}{*}{ Rotational Direction } & Relation of $m_{\mathbf{1}}$ with $\boldsymbol{m}_{\mathbf{2}}$ & \multicolumn{2}{c}{ Judgment Results } \\
\cline { 2 - 4 } & & Location of the Rotational Center $\boldsymbol{O}$ & Sign of $\boldsymbol{\omega}$ \\
\hline \multirow{3}{*}{ Clockwise } & $m_{1}>0, m_{2}<0$ & Between LVS1 and LVS2 & + \\
& $0<m_{1}<m_{2}$ & Below both LVS1 and LVS2 & + \\
& $m_{1}<m_{2}<0$ & Above both LVS1 and LVS2 & + \\
\hline \multirow{2}{*}{ Counter-clockwise } & $m_{1}<0, m_{2}>0$ & Between LVS1 and LVS2 & - \\
& $0>m_{1}>m_{2}$ & Below both LVS1 and LVS2 & - \\
& $m_{1}>m_{2}>0$ & Above both LVS1 and LVS2 & - \\
\hline
\end{tabular}

\section{Experimental Calculations}

The rotation of a planar wooden disk with rough surface is taken as an example to confirm the validity of the proposed method for remotely sensing rotational velocity. The confirmation can be realized according to the following steps: (1) The wooden disk is installed on the shaft of a DC-micromotor (Model 2230G0003, Faulhaber GmbH \& Co. KG, Schönaich, Germany) which drives the rotation of the disk. The angular speed of the DC-micromotor is controlled by the output voltage of an adjustable DC regulated power supply (Model APS3005S-3D, Atten Technology Co., Shenzhen, China); (2) The rotational velocity of the wooden disk is set by adjusting the output voltage and then measuring the rotational velocity with a DT-2234B Digital Tachometer (Suwei Co. Ltd., Guangzhou, China); (3) Sunlight or very bright white LEDs illuminate the wooden disk whose surface is imaged on a QPD through a telecentric imaging system (telescope) with magnification of $1 \times$. The telecentric imaging system includes an adjustable aperture and two composite objectives with a focal length of $\sim 26 \mathrm{~mm}$. The QPD is constructed by four identical photovoltaic-cells with the active area of $0.89 \times 4.39 \mathrm{~mm}^{2}$, where two photovoltaic cells of each linear velocity sensor (LVS1/LVS2) directly come from two adjacent pixels of a Si-based photovoltaic-cell array (Model A5V-38, OSI Optoelectronics Inc., Hawthorne, CA, USA), and then the LVS1 and LVS2 form the QPD. Other parameters of the QPD are as follows: $P=0.99 \mathrm{~mm}$ and $L=16 \mathrm{~mm}$; (4) The output signals of the QPD are amplified and then converted into discrete data by four A/D converters with the sampling interval of $T_{0}=0.2 \mathrm{~ms}$. The amplifier and the A/D converter are INA128 (Texas Instruments Inc., Dallas, TX, USA) and AD7663 (Analog Devices Inc., Norwood, MA, USA) with 16-bit resolution, respectively; (5) The discrete data are sent to a DPS, in which $\Phi_{1}(m)$ and $\Phi_{2}(m)$ are calculated, and their minimums are searched to get 
$m_{1}$ and $m_{2}$. Then the rotational velocity $\omega$ is calculated with Equation (12); (6) Finally, we compare the calculated value and direction to the given ones, and judge whether they are agree with each other.

When the wooden disk was rotated at a rotational velocity of $6.3 \mathrm{rad} / \mathrm{s}$ in the clockwise direction, we located the rotational center $O$, whose distance to its closest LVS was $\sim 6 \mathrm{~mm}$, to be between the LVS1 and the LVS2. The output signals $S_{1}(t), S_{2}(t), S_{3}(t)$ and $S_{4}(t)$ of the QPD are shown in Figure $7 \mathrm{a}, \mathrm{b}, \mathrm{d}, \mathrm{e}$, respectively. Figure $7 \mathrm{c}, \mathrm{f}$ illustrate the calculated $\Phi_{1}(m)$ and $\Phi_{2}(m)$, respectively. The minima of $\Phi_{1}(m)$ and $\Phi_{2}(m)$ were searched at $m_{1}=79$ and $m_{2}=-131$, respectively. According to Equation (12), the calculated rotational velocity was $6.278 \mathrm{rad} / \mathrm{s}$ with the " + " sign that represents the clockwise direction. These results demonstrate that the calculated value and direction of the rotational velocity agree with the given ones.

When the wooden disk rotated counter-clockwise at a rotational velocity of $1.9 \mathrm{rad} / \mathrm{s}$, we let the rotational center $O$, whose interval to its nearest LVS was $\sim 10 \mathrm{~mm}$, be below both the LVS1 and the LVS2. The output signals $S_{1}(t), S_{2}(t), S_{3}(t)$ and $S_{4}(t)$ are exhibited in Figure $8 \mathrm{a}, \mathrm{b}, \mathrm{d}$,e, respectively. Figure $8 \mathrm{c}, \mathrm{f}$ plot the calculated DSCs $\Phi_{1}(m)$ and $\Phi_{2}(m)$, respectively, from which we derived $m_{1}=-100$ and $m_{2}=-260$. Based on Equation (12), the calculated rotational velocity was $-1.904 \mathrm{rad} / \mathrm{s}$ whose " - " sign indicates the counter-clockwise direction. The calculated rotational velocity including its amplitude and direction is also in agreement with the given one. Similarly, we performed the corresponding experimental calculations in the cases of other rotational directions and center locations, where the calculated rotational velocities were also in agreement with their given ones.

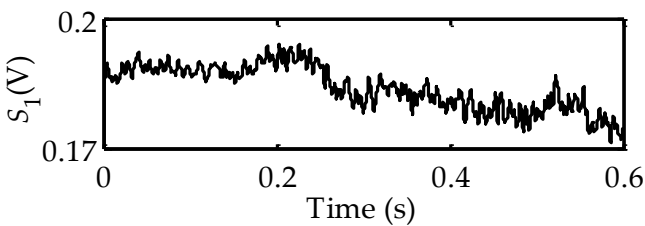

(a)

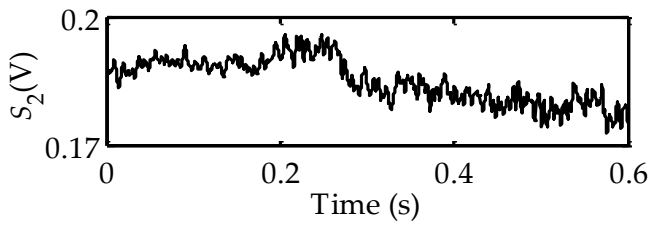

(b)

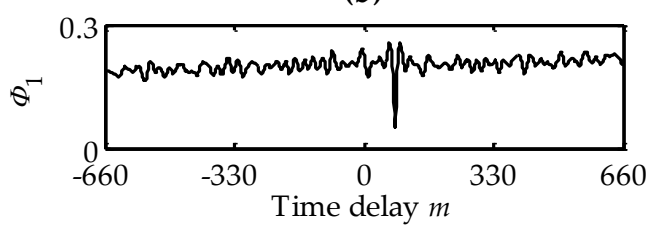

(c)

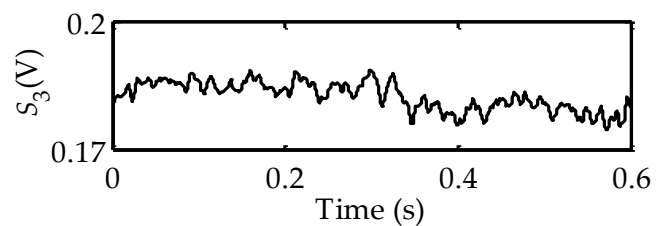

(d)

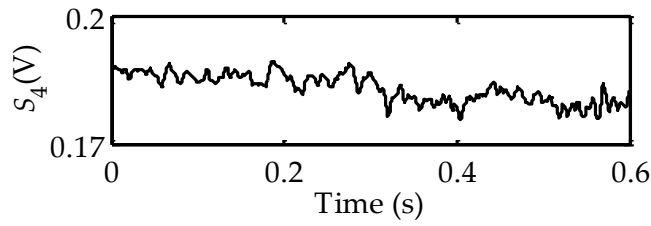

(e)

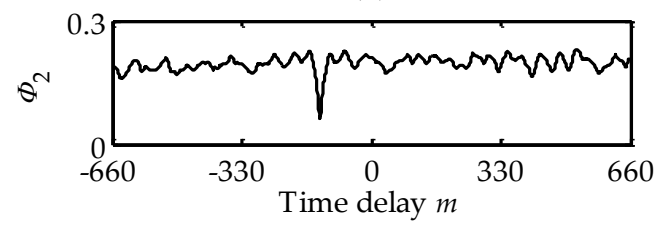

(f)

Figure 7. DSCs in the cases of clockwise rotation and the rotational center locating between the LVS1 and the LVS2: $(\mathbf{a}, \mathbf{b}, \mathbf{d}, \mathbf{e})$ are output signals of the QPD; (c) time delay between signals $S_{1}$ and $S_{2} ;(\mathbf{f})$ time delay between signals $S_{3}$ and $S_{4}$.

The abovementioned calculations had a relative amplitude error of $\sim 0.3 \%$. Their resolutions were up to $0.011 \mathrm{rad} / \mathrm{s}$, and their optimal resolution was $5.4 \times 10^{-5} \mathrm{rad} / \mathrm{s}(N=4800)$. The resolution and its optimum of the DSC-based rotational velocity are actually determined by $P /\left(L T_{0} m_{1} m_{2}\right)$ and $4 P /\left(L T_{0} \mathrm{~N}^{2}\right)$, respectively, which are related to the sampling interval $T_{0}$ and the rotational velocity. For measuring super-low rotational velocities, the resolution can be improved to be excellent by increasing the sampling interval in the case of guaranteeing enough precision. In other often used methods, the resolution of laser gyroscopes can reach $0.01^{\circ} / \mathrm{h} \sim 10^{\circ} / \mathrm{h}$ [4], and the TCC-based method has a relative error of $3.33 \%$ [12]. The marking method with an optical label plus quadrant photodiode possesses a relative error of $0.001 \%$ [24], while it measures only the average rotational velocity within 
the angular displacement of $2 \pi \mathrm{rad}$. The circular-grating-based methods with Moire optical grating and capacitive grating have relative errors of $\sim 0.07 \%$ and $0.44 \%$, respectively [30]. Compared to the existing method, the DSC-based method proposed has mid-ranking resolution and accuracy, whereas on the plus side, it does not require installing any standard division disk.

To verify the computational speed, the DSC-based and TCC-based calculations were performed in an Acer PC system equipped with an Intel dual-core i3-processer plus $3.4 \mathrm{GHz}$, OS Windows 10 and Matlab 2010. The DSC-based calculations needed average times of $141 \mathrm{~ms}$ and $86 \mathrm{~ms}$ in the cases of $N=4800$ and $N=3600$ data numbers, respectively, and the TCC-based ones consumed on average times of $158.92 \mathrm{~s}$ and $72.849 \mathrm{~s}$, correspondingly. These achievements imply that the DSC-based computational speed has been improved up to 1127 times that of the TCC-based one in the case of a data number $N>4800$. Thus the DSC-based calculation for rotational velocity is very fast and accurate, and is not affected by the movement and alignment error of the QPD relative to the measured object, making it fit for remotely evaluating transient angular-velocities. These features are due to the following reasons: (1) $\left(1 / m_{1}-1 / m_{2}\right)$ always makes $\left|R_{1} \pm R_{2}\right|$ equal to constant $L$, in the cases of arbitrary rotational center, eccentricity, radial fluctuation or parallel movement; (2) the amplification coefficient $M$ has been hidden in $R_{1}$ and $R_{2}$ on the image plane, which leads to the insensitivity to various $M$ changed by alignment error and axial shaking; (3) $V_{1} \pm V_{2}$ is always not related to eccentricity, parallel movement and radial shift. Thus the rotational velocity calculated with Equation (12) is only determined by pure rotation, which has much fewer error sources.

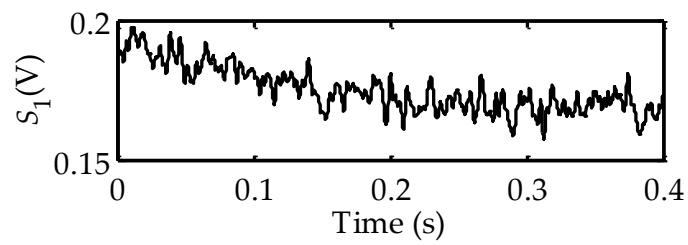

(a)

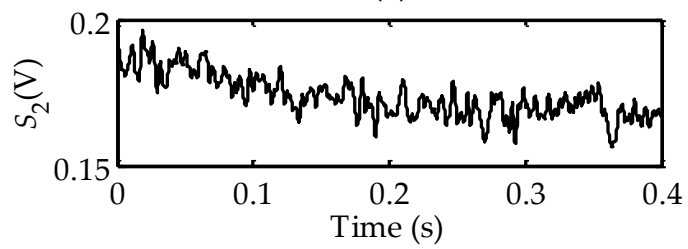

(b)

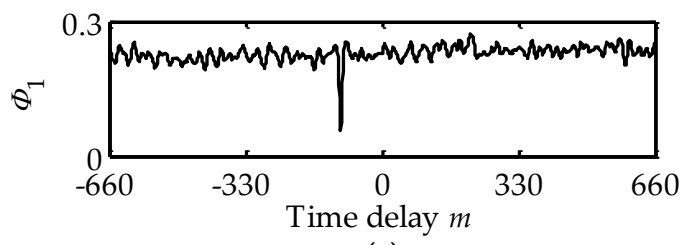

(c)

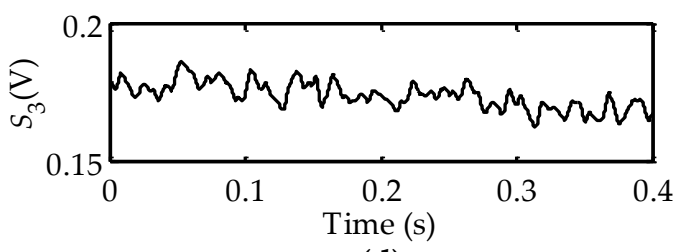

(d)

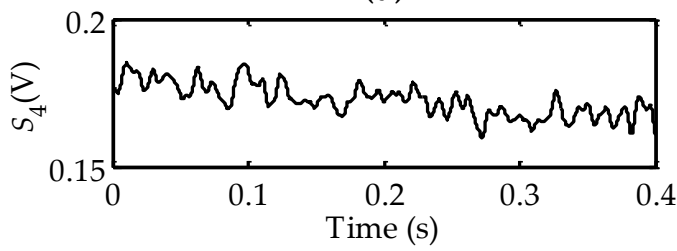

(e)

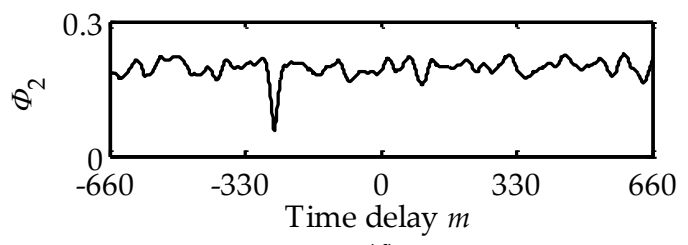

(f)

Figure 8. DSCs in the cases of the counter-clockwise rotation and the rotational center locating below both the LVS1 and the LVS2: (a, b, d,e) are output signals of the QPD; (c) time delay between signals $S_{1}$ and $S_{2} ;(\mathbf{f})$ time delay between signals $S_{3}$ and $S_{4}$.

\section{Conclusions}

The method of remotely evaluating rotational velocity has been presented in this paper, where the rotational velocity is derived from the temporally-delayed serial-numbers at the minima of two DSCs that are calculated with the four stochastic output signals of the QPD formed by four identical photovoltaic-cells, and the sign of the rotational velocity represents the rotational direction. The DSC algorithm is implemented by differentiating and subtracting the random output signals of two photovoltaic cells, and then integrating the absolute value of the differential subtraction signal. In the DSC algorithm, the rough surface and random reflection of measured object will 
have a contribution to precisely determining the temporal delay and rotational velocity, owing to its difference operation. Experimental calculations were performed to confirm the proposed evaluation method. The calculated rotational velocities, including their amplitudes and directions, were in excellent agreement with their given ones, which possessed an amplitude error of $\sim 0.3 \%$, and had over 1100 times the TCC-based efficiency in the case of data number $N>4800$.

The proposed evaluation method works without any circular indexing plate, and its DSC does not require any multiplication or division operations. At the same time, this method is insensitive to the radial fluctuation, axial shift, eccentricity and parallel movement of the measured object that has assembly or mismatch errors. The rotational velocity calculated with Equation (12) is only determined by pure rotation, which has many fewer error sources. Comparing to the SF method, this evaluation method does not require calculating central frequencies, center position and extra direction, and it can simultaneously determine the amplitude and direction of a measured rotational velocity. In general, the proposed method is simple, fast, accurate and effective for remotely evaluating transient rotational velocities.

Acknowledgments: This work was supported partially by the National Natural Science Foundation of China (Grant No. 61575035 and 51304260), and also by the Chongqing Key Laboratory of Time-grating Sensing and Advanced Test Technology, China (Grant No. 2013TGS006).

Author Contributions: This paper is completed by the listed authors. Xiangkai Zeng contributed to the theoretical analysis, principle and proofs, and supervised the work and helped with editing and polishing the manuscript; Zhixiong Zhu wrote the paper, contributed to the confirmation experiments, and took part in the theoretical proofs; Yang Chen participated in the programming. All authors of the article provided substantive comments.

Conflicts of Interest: The authors declare no conflict of interest.

\section{References}

1. Ciminelli, C.; Dell'Olio, F.; Campanella, C.E.; Armenise, M.N. Photonic technologies for angular velocity sensing. Adv. Opt. Photonics 2010, 2, 370-404. [CrossRef]

2. Zheng, S.; Han, B. Investigations of an integrated angular velocity measurement and attitude control system for spacecraft using magnetically suspended double-gimbal CMGs. Adv. Space Res. 2013, 51, 2216-2228. [CrossRef]

3. Dell'Olio, F.; Tatoli, T.; Ciminelli, C.; Armenise, M.N. Recent advances in miniaturized optical gyroscopes. J. Eur. Opt. Soc. Rapid Publ. 2014, 9, 14013. [CrossRef]

4. Ciminelli, C.; D’Agostino, D.; Carnicella, G.; Dell'Olio, F.; Conteduca, D.; Ambrosius, H.P.M.; Smit, M.K.; Armenise, M.N. A high-Q InP resonant angular velocity sensor for a monolithicaly integrated optical gyroscope. IEEE Photonics J. 2016, 8, 6800418. [CrossRef]

5. Ciminelli, C.; Dell'Olio, F.; Campanella, C.E.; Armenise, M.N. Numerical and experimental investigation of an optical high-Q spiral resonator gyroscope. In Proceedings of the IEEE Conference on Transparent Optical Networks(ICTON), Coventry, UK, 2-5 July 2012; pp. 1-4.

6. Campanella, C.E.; de Leonardis, F.; Passaro, V.M.N. Fiber Bragg grating ring resonators under rotation for angular velocity sensing. Appl. Opt. 2015, 54, 4789-4796. [CrossRef] [PubMed]

7. Lin, C.E.; Yu, C.J.; Chen, C.C. Design of a full-dynamic-range balanced detection heterodyne gyroscope with common-path configuration. Opt. Express 2013, 21, 9947-9958. [CrossRef] [PubMed]

8. Zhang, Y.; Guo, Y.; Li, C.; Wang, Y.; Wang, Z. A New Open-Loop Fiber Optic Gyro Error Compensation Method Based on Angular Velocity Error Modeling. Sensors 2015, 15, 4899-4912.

9. Lin, S.T.; Yeh, S.L.; Lin, Z.F. Angular probe based on using Fabry-Perot etalon and scanning technique. Opt. Express 2010, 18, 1794-1800. [CrossRef] [PubMed]

10. Zheng, D.; Wang, X.; Sasaki, O. Parallel plate interferometer with a reflecting mirror for measuring angular displacement. Opt. Rev. 2007, 14, 314-318. [CrossRef]

11. Li, B.; Jiang, L.; Wang, S.; Zhou, L.; Xiao, H.; Tsai, H.L. Ultra-abrupt tapered fiber Mach-Zehnder interferometer sensors. Sensors 2011, 11, 5729-5739. [CrossRef] [PubMed]

12. Nanzer, J. Millimeter-wave interferometric angular velocity detection. IEEE Trans. Microw. Theory Tech. 2010, 58, 4128-4136. [CrossRef] 
13. Zhong, Z.; Sun, J. Angular-displacement measurement with the digital-speckle correlation method. In Proceedings of the IEEE Conference on Multimedia Technology, Hangzhou, China, 26-28 July 2011.

14. Tendela, L.P.; Galizzi, G.E.; Federico, A.; Kaufmann, G.H. Measurement of nanometric displacements by correlating two speckle interferograms. Appl. Opt. 2011, 50, 1758-1764. [CrossRef] [PubMed]

15. Hanus, R.; Petryka, L.; Zych, M. Velocity measurement of the liquid-solid flow in a vertical pipeline using gamma-ray absorption and weighted cross-correlation. Flow Meas. Instrum. 2014, 40, 58-63. [CrossRef]

16. Seraj, H.; Rahmat, M.F.; Khalid, M. Measurement of velocity of solid/air two phase fluid using electrostatic sensors and cross correlation technique. Sci. Iran. 2013, 20, 786-792. [CrossRef]

17. Gajewski, J.B. Accuracy of cross correlation velocity measurements in two-phase gas-solid flows. Flow Meas. Instrum. 2013, 30, 133-137. [CrossRef]

18. Seo, K.W.; Lee, S.J. High-accuracy measurement of depth-displacement using a focus function and its cross-correlation in holographic PTV. Opt. Express 2014, 22, 15542-15553. [CrossRef] [PubMed]

19. Heid, T.; Kääb, A. Evaluation of existing image matching methods for deriving glacier surface displacements globally from optical satellite imagery. Remote Sens. Environ. 2012, 118, 339-355. [CrossRef]

20. Debella-Gilo, M.; Kääb, A. Sub-pixel precision image matching for measuring surface displacements on mass movements using normalized cross-correlation. Remote Sens. Environ. 2011, 115, 130-142. [CrossRef]

21. Mauger, C.; Méès, L.; Michard, M.; Lance, M. Velocity measurements based on shadowgraph-like image correlations in a cavitating micro-channel flow. Int. J. Multiph. Flow 2014, 58, 301-312. [CrossRef]

22. Fasano, G.; Rufino, G.; Accardo, D.; Grassi, M. Satellite angular velocity estimation based on star images and optical flow techniques. Sensors 2013, 13, 12771-12793.

23. Yang, Y.P.; Ting, Y.Y. Improved angular displacement estimation based on hall-effect sensors for driving a brushless permanent-magnet motor. IEEE Trans. Ind. Electron. 2014, 61, 504-511. [CrossRef]

24. Qian, J.; Cui, Y.; Xu, P. The study for measuring rotor speed and direction with quadrant photoelectric detector. Measurement 2008, 41, 626-630. [CrossRef]

25. Lay, Y.L.; Chen, W.Y. Rotation measurement using a circular moiré grating. Opt. Laser Technol. 1998, 30, 539-544. [CrossRef]

26. Yu, Y.; Wang, S.; Li, B.; Wu, H.; Cao, Z. High resolution angular-displacement sensor based on whispering gallery mode resonance in bent optical fibers. J. Beijing Inst. Technol. 2014, 23, 42-46.

27. Hao, S.; Liu, J.; Hao, M.; Song, B. Design of High Precision Magnetic Grid Displacement Sensor. In Proceedings of the IEEE Conference on Mechatronics and Automation, Takamatsu, Japan, 5-8 August 2008; pp. 185-188.

28. Gao, Z.; Chen, X.; Peng, D. Online self-calibration system for time grating angular displacement sensor. Opt. Precis. Eng. 2015, 23, 93-101.

29. Zeng, X.K.; Chen, Y.; Zhu, Z.X.; Liu, Q.S. Identification method of rotational direction in remote angular velocity measurement with spatial filtering technique. Acta Opt. Sin. 2015, 35, 0612001. [CrossRef]

30. Lu, Z.; Pei, D.X.; Bai, R.; Liu, L. Design of rotating speed calibrating device of capacitive sensor based on FPGA. Electro-Opt. Technol. Appl. 2015, 30, 38-41.

(C) 2016 by the authors; licensee MDPI, Basel, Switzerland. This article is an open access article distributed under the terms and conditions of the Creative Commons Attribution (CC-BY) license (http://creativecommons.org/licenses/by/4.0/). 